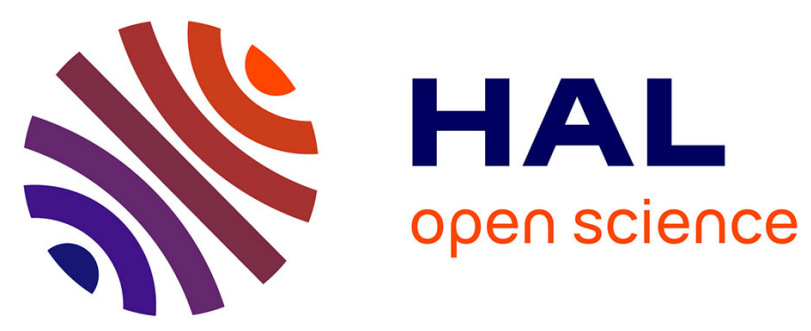

\title{
3TR: a pan-European cross-disease research consortium aimed at improving personalised biological treatment of asthma and COPD
}

Celeste Porsbjerg, Anke Maitland-van Der Zee, Guy Brusselle, Giorgio Walter Canonica, Alvar Agusti, Rosa Faner, Claus Vogelmeier, Martijn Nawijn, Maarten van den Berge, Franca Rusconi, et al.

\section{To cite this version:}

Celeste Porsbjerg, Anke Maitland-van Der Zee, Guy Brusselle, Giorgio Walter Canonica, Alvar Agusti, et al.. 3TR: a pan-European cross-disease research consortium aimed at improving personalised biological treatment of asthma and COPD. European Respiratory Journal, 2021, 58 (4), pp.2102168. 10.1183/13993003.02168-2021 . hal-03403437

\section{HAL Id: hal-03403437 \\ https://hal.science/hal-03403437}

Submitted on 26 Oct 2021

HAL is a multi-disciplinary open access archive for the deposit and dissemination of scientific research documents, whether they are published or not. The documents may come from teaching and research institutions in France or abroad, or from public or private research centers.
L'archive ouverte pluridisciplinaire HAL, est destinée au dépôt et à la diffusion de documents scientifiques de niveau recherche, publiés ou non, émanant des établissements d'enseignement et de recherche français ou étrangers, des laboratoires publics ou privés. 


\section{TR: a pan-European cross-disease research consortium aimed at improving personalised biological treatment of asthma and COPD}

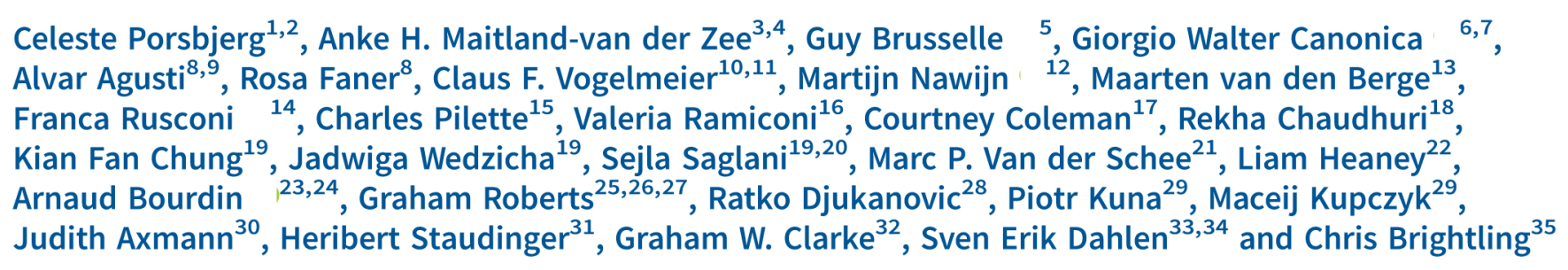

${ }^{1}$ Dept of Respiratory Medicine, Bispebjerg University Hospital, Copenhagen, Denmark. ${ }^{2}$ Copenhagen Center for Translational Research, Bispebjerg Hospital, Copenhagen, Denmark. ${ }^{3}$ Dept of Respiratory Medicine, Amsterdam UMC, University of Amsterdam, Amsterdam, The Netherlands. ${ }^{4}$ Dept of Pediatric Pulmonology, Amsterdam UMC, University of Amsterdam, Amsterdam, The Netherlands. ${ }^{5}$ Dept of Respiratory Medicine, Ghent University Hospital, Ghent, Belgium. ${ }^{6}$ Personalized Medicine, Asthma and Allergy Humanitas Clinical and Research Center IRCCS, Rozzano, Italy. ${ }^{7}$ Dept of Biomedical Sciences, Humanitas University, Pieve Emanuele, Italy. ${ }^{8}$ Hospital Clinic, University of Barcelona, Barcelona, Spain. ${ }^{9}$ IDIBAPS, CIBERES, Barcelona, Spain. ${ }^{10}$ Dept of Medicine, Pulmonary and Critical Care Medicine, University of Marburg (UMR), Marburg, Germany. ${ }^{11}$ Member of the German Center for Lung Research (DZL), Marburg, Germany. ${ }^{12}$ Dept of Pathology and Medical Biology, University of Groningen, University Medical Center Groningen, GRIAC Research Institute, Groningen, The Netherlands. ${ }^{13}$ Dept of Pulmonary Diseases, University of Groningen, University Medical Center Groningen, Groningen, The Netherlands. ${ }^{14}$ Unit of Epidemiology, Meyer Children's University Hospital, Florence, Italy. ${ }^{15}$ Dept of pneumology, Cliniques universitaires Saint-Luc and Institute of Experimental and Clinical Research, Université catholique de Louvain, Brussels, Belgium. ${ }^{16}$ European Federation of Allergy and Airways Diseases Patients' Associations (EFA), Brussels, Belgium. ${ }^{17}$ European Lung Foundation, Sheffield, UK. ${ }^{18}$ Division of Infection, Immunity and Inflammation, University of Glasgow, Glasgow, UK. ${ }^{19}$ National Heart and Lung Institute, Imperial College London, London, UK. ${ }^{20}$ Dept of Paediatric Respiratory Medicine, Royal Brompton Hospital, London, UK. ${ }^{21}$ Owlstone Medical Ltd, Cambridge, UK. ${ }^{22}$ Wellcome-Wolfson Centre for Experimental Medicine, School of Medicine, Dentistry and Biomedical Sciences, Queen's University, Belfast, UK. ${ }^{23}$ Dept of Respiratory Diseases, Univ Montpellier, CHU Montpellier, Montpellier, France. ${ }^{24}$ PhyMedExp, Univ Montpellier, CNRS, INSERM, Montpellier, France. ${ }^{25}$ Faculty of Medicine, University of Southampton, Southampton, UK. ${ }^{26}$ NIHR Southampton Biomedical Research Centre, University Hospital Southampton NHS Foundation Trust, Southampton, UK. ${ }^{27}$ The David Hide Asthma and Allergy Research Centre, St Mary's Hospital, Isle of Wight, UK. ${ }^{28}$ Clinical and Experimental Science, Faculty of Medicine and NIHR Southampton Biomedical Research centre, Southampton, UK. ${ }^{29}$ Dept of Internal Medicine, Asthma and Allergy, Medical University of Lodz, Lodz, Poland. ${ }^{30}$ Immunology, Infectious Diseases and Ophthalmology Discovery and Translational Area, Roche Pharma Research \& Early Development, F. Hoffmann-La Roche Ltd, Basel, Switzerland. ${ }^{31}$ Clinical Strategy Lead, Immunology and Inflammation Therapeutic Area, Sanofi Genzyme, Bridgewater, NJ, USA. ${ }^{32}$ Translational Science and Experimental Medicine, Research and Early Development, Respiratory \& Immunology, BioPharmaceuticals R\&D, AstraZeneca, Gothenburg, Sweden. ${ }^{33}$ The Center for Allergy Research and the Institute of Environmental Medicine, Karolinska Institutet, Stockholm, Sweden. ${ }^{34}$ Dept of Respiratory Medicine, Karolinska University Hospital Huddinge, Stockholm, Sweden. ${ }^{35}$ Dept of Respiratory Sciences, University of Leicester, Leicester, UK.

Corresponding author: Celeste Porsbjerg (celeste.porsbjerg@regionh.dk) 
systemic lupus erythematosus, rheumatoid arthritis, multiple sclerosis, ulcerative colitis and Crohn's disease.

Asthma and COPD are some of the most common chronic diseases; there are approximately 600 million people with asthma and 400 million with COPD worldwide [1, 2], with COPD being the third leading cause of death worldwide [2].

Management options of both asthma and COPD have advanced significantly, with both new inhaler formulation and biological treatments that target specific disease pathways transforming treatment options for patients with severe asthma [3]. However, these treatments do not completely abolish all exacerbations or symptoms, they do not achieve complete disease control and they do not cure the disease. In addition, they are directed against type 2 inflammation, but no treatments are so far available for patients without type 2, or for patients with COPD. Therefore, there is a further need for improved targeting of biological (and other) treatments.

A range of biological treatments developed for asthma have been tested in COPD, with limited efficacy; a clearer understanding of immunological disease drivers in COPD is necessary. Although there are commonalities between asthma and COPD, such as eosinophilia being a predictor of exacerbations and responsiveness to inhaled corticosteroids, there are also clear differences, illustrated by the modest effects of blocking IL-5 in COPD patients with high levels of eosinophils, compared to their marked pronounced beneficial effects in severe eosinophilic asthma [4, 5].

Both asthma and COPD are chronic conditions, which require continuous, possibly life-long, treatment. No disease-modifying treatments exist at this point (except allergen immunotherapy in very selected cases [6]), and there is a need for increasing treatment ambitions, to aim for remission of disease [7]. The ambition of 3TR is to move the field of immune-modifying treatments forward in a pan-European, concerted effort by combining the forces of clinical researchers and scientists in a 7-year collaborative effort.

Specifically, the focus of the 3TR consortium is to address:

- What specifically constitutes a response or non-response to biological therapies in asthma and COPD?

- What are the underlying immunological mechanisms that explain the reasons for response versus non-response? And for super-response versus response?

- Can we identify biomarker profiles predicting response or non-response by combining repeated bio-sampling from multiple compartments with advanced multi-omics methods in patients treated with biologics?

- What can we learn from comparing different immune-mediated diseases across different organs and systems?

- How can we translate these insights into better approaches for precision medicine for asthma and COPD, such as the introduction of new biomarkers?

The consortium involves a collaboration between academic researchers, patients and the pharmaceutical industry.

\section{Potentials for developing solutions}

The most significant advances in immune-targeted therapies have occurred in asthma, potentially ultimately paving the way towards such therapies for COPD. At present, the advent of biological treatments for severe asthma represents a unique opportunity to further unravel the pathogenesis of obstructive airway diseases. Specifically, a lack of response to biologics, as well as a super-response, may provide important insight into pivotal immunological mechanisms and pave the way towards more effective interventions.

As a first important focus, we need to have a clearer understanding and agreed concept of what qualifies as a response: a number of response outcomes are of importance, including patient-reported outcomes, exacerbation rates and lung function, and prevention of disease progression, which should be achieved with minimal treatment side effects. Currently, biomarkers such as eosinophils and exhaled nitric oxide fraction may be used to predict a response to $\mathrm{T} 2$ biologics, but more alternatives and especially non-T2 markers are needed. Different definitions of response and non-response have been proposed [8, 9], but it remains uncertain how specific response profiles translate into long-term outcomes: is complete abrogation of exacerbations necessary for patients to achieve long-term complete clinical control of their disease? Is complete suppression of disease signatures, such as all T2 biomarkers, important? 
A second important focus is to link specific immune mechanisms to either a non-response or a "super-response" (i.e. above what could be expected based on results from phase 3 trials), or to "clinical remission" to identify future treatment targets. Specifically, a future ambition is to aim for remission of disease as a management goal: achieving long-term complete control and preventing progression of the disease [7]. Within rheumatology, treating with the aim to achieve complete clinical control has translated into better remission rates $[10,11]$. A similar effect may be seen in severe asthma where a complete control of both symptoms and airway inflammation could potentially improve the likelihood of long-term control. By furthering our understanding of the immunological mechanisms driving eosinophilic COPD, we may also move towards better management strategies for inducing long-term stability and prevention of progression in patients with COPD. A unique aspect of 3TR is precisely the cross-disease focus that enables evaluation of immunological pathways associated with disease controls and remission, irrespective of diagnostic label.

A third focus of 3TR is the potential of advanced immunological analysis methods: molecular profiling and integration of multi-omics data may stratify individuals with clinically heterogeneous diseases to identify the molecular basis of their pathogenesis and potential new biomarkers. Through large-scale research consortia such as U-BIOPRED, we have achieved important insights into the heterogeneity of severe asthma that are likely to direct the way towards better management strategies [12, 13]. However, most existing studies have so far been cross-sectional or without specific immune-modifying interventions.

With the advent of a range of targeted biological treatments for treating severe asthma, we are now in a position to study the immunological effects of blocking individual immune pathways, such as IgE, IL-5 and IL-4/13, and the upstream TSLP and IL33 pathways, to gain better insights into the pathogenetic drivers of severe asthma. This mandates longitudinal studies with biosampling "real-life" patients treated with biologics.

For COPD, studies targeting the same pathways will enable us to compare mechanisms across asthma and COPD, and to move forward our understanding of similarities and dissimilarities between the two conditions. As the effect of targeted monoclonal antibodies has been less convincing in COPD in general, there is an urgent the need to determine the mechanisms involved in a clinical response. By applying advanced immunological analyses to samples obtained before and after specific antibody treatments, and comparing responders to non-responders, we may obtain a clearer understanding of key pathogenic mechanisms, to aid in developing more effective therapies.

How to unleash these potentials: 3TR

The strategy of 3TR combines five key elements: 1) the expertise of world-leading experts, clinical researchers and translational scientists in each disease area, with pharmaceutical companies, patient organisations, and small- and medium-sized enterprises; 2) prospective collection of multiple samples from both diseased tissue and blood; 3) use of samples from existing studies; 4) advanced state-of-the-art multi-omics analysis; and, finally, 5) use of a "carousel model" to boost the efficient generation of evidence throughout the 7-year duration of 3TR, allowing a simultaneous and continuous process of analysing existing data and samples, alongside collecting new samples (figure 1).

As a primary step, to define therapeutic response and non-response and to allow comparisons across different studies, it is necessary to study biomarkers and immunological mechanisms related to different responder profiles. A key aim of the 3TR Asthma and COPD Work Package (3TR WP8) is, therefore, to map and define outcome measures for documenting therapeutic response. These outcomes will take into consideration the opinions of the three stakeholders of 3TR: patients (may rather focus on the ability to lead a normal daily life), clinicians (likely to focus on objective outcomes) and healthcare funders (likely to focus on healthcare costs). Ultimately, criteria for non-response and response for use in future trials will be defined in clinical practice.

In order to validate biomarkers predicting response, existing studies will be used in parallel with introduction of de novo studies, in the carousel model proposed by 3TR WP8. Within the respiratory field, a number of phase 2 and 3 trials are ongoing or will be soon started, examining the effects of monoclonal antibody treatment in patients with asthma and COPD. The participation of industry partners in 3TR represents a forceful opportunity to study and compare the effects of targeting individual immune pathways in asthma and COPD. Simultaneously, large multicentre academia-led trials are studying parallel questions on the effects of biologics, such as the Predictumab trial comparing the effect of anti-IgE to anti-IL5 in severe asthma patients eligible for both treatments, or the BenRex trial, studying exacerbations of severe asthma during treatment with benralizumab. Observations in these studies will be combined with those in 


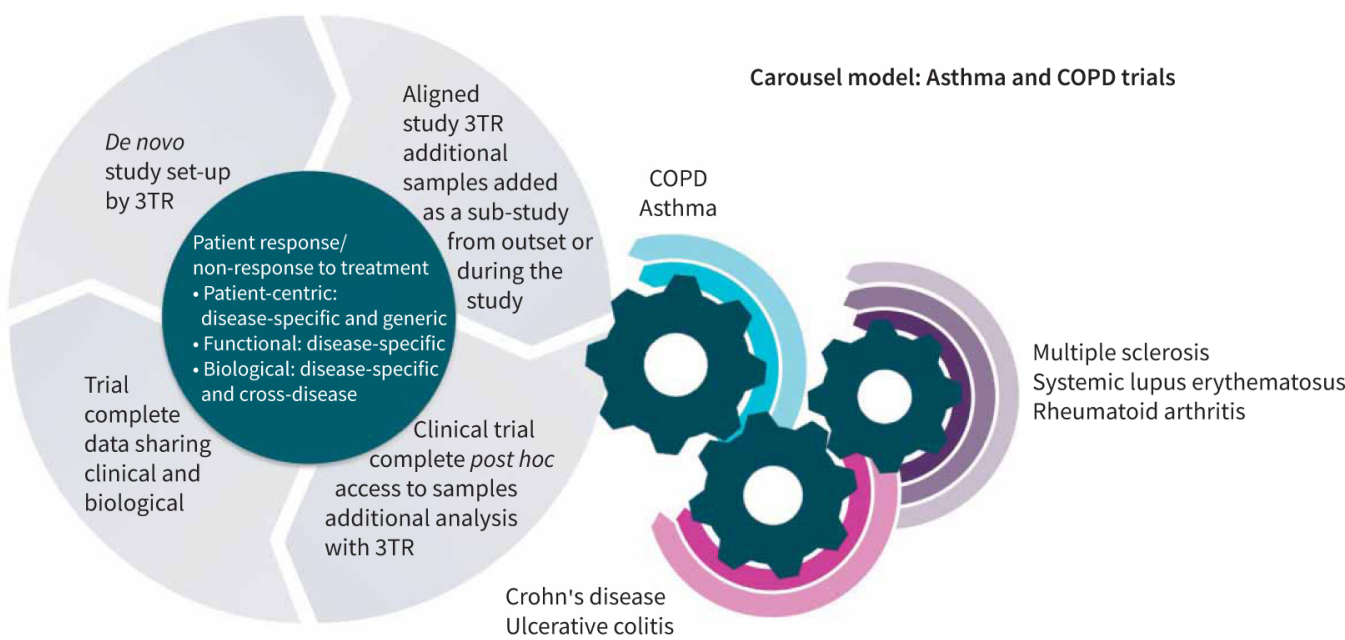

FIGURE 1 The 3TR carousel model: samples obtained from existing studies are analysed in parallel with prospective sampling in aligned and de novo studies, to achieve effective generation of data throughout the 7-year duration of the 3TR programme.

the SoMOSA (Study of Mechanisms of action of Omalizumab in Severe Asthma), identifying biomarkers predicting reductions in exacerbation rates and use of oral corticosteroids.

WP8 will set up a trial platform to enable cross-study analyses, providing an overview of studies with a catalogue of existing and planned studies and enabling cross-study analyses by defining common standard operating procedures (SOPs) for setting up de novo cohorts, outlining study visits, clinical assessments, sample types and sample processing.

Additionally, WP8 will set up a pan-European prospective cohort, to assess and compare responses to different biological treatments in a large real-life population of patients with severe asthma, including both adults and children. By comparing responders and non-responders, the 3TR Asthma Biologics Cohort study (3TR-ABC) will describe immunological mechanisms related to response profiles and identify biomarkers for predicting response, as well as possible future treatment targets (figure 2).

As biological therapies are not yet approved for COPD, samples from pharmaceutical trials will be used to investigate immune mechanisms involved in response versus non-response. The 3TR-ABC study will be followed by the establishment of a cohort of COPD patients, which may ultimately include patients commenced on a biological treatment, if such treatments are approved for clinical use.

Obtaining high-quality airway samples will be key to successful multi-omics analysis, highlighted by the experience from previous translational studies such as U-BIOPRED, in which a significant proportion of sputum samples were not of adequate quality for performing omics analysis [12]. A catalogue of SOPs describing the processing of bio-samples will be assembled, combining SOPS from previous studies with novel sampling methods and protocol optimisation.

Finally, by including assessment of other immune-mediated diseases, the 3TR-ABC study enables exploration of shared immune pathways across different diseases, providing new insights into asthma and COPD as systemic diseases, and their potential endotypic connections with other non-respiratory immune diseases.

\section{Perspectives}

The insights into immune disease mechanisms in asthma and COPD provided by 3TR may uncover future treatment targets and identify relevant patient populations to address such targets. Furthermore, the validation of biomarkers to guide management choices is increasingly important, considering the increasing numbers of biologics available. Finally, as we move towards higher ambitions for treatment outcomes in asthma and COPD, the work undertaken by 3TR will provide an important basis for future studies on disease modification. 

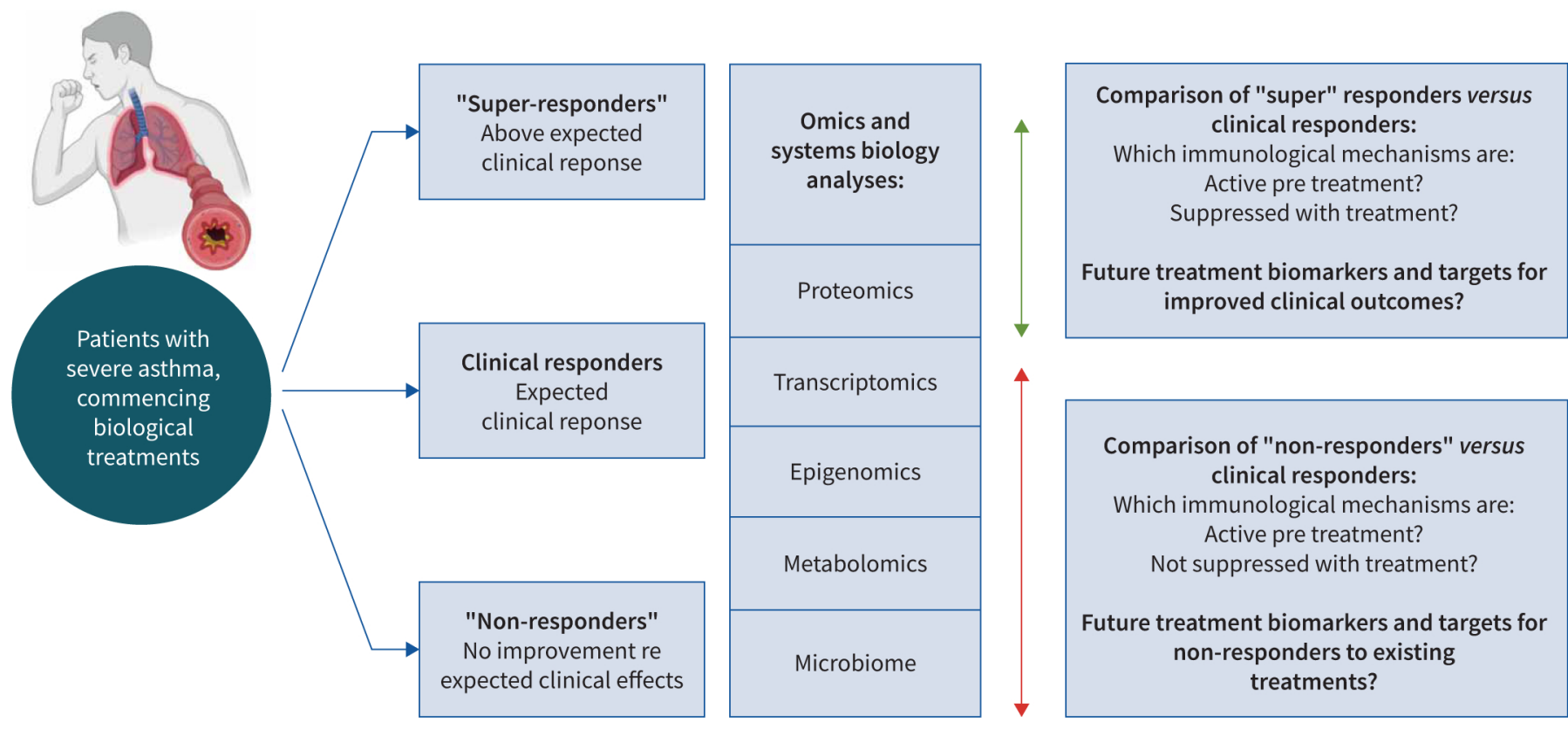

FIGURE 2 The 3TR Asthma Biologics Study (3TR-ABC): identifying novel treatment targets in severe asthma through the response to biological therapy.

In conclusion, the 3TR research consortium brings together European, patients, pharma and other stakeholders or experts in different medical specialties to elucidate disease mechanisms in this unique era of targeted biologics, unleashing strong potentials ultimately to move towards more ambitious treatment goals for patients with asthma and COPD through precision medicine in our field of interest and, potentially, also in other medical subspecialties.

Conflict of interest: C. Porsbjerg declares grants or contracts, consulting fees and payment or honoraria from AstraZeneca, GlaxoSmithKline, Novartis, TEVA, Sanofi, Chiesi and ALK in the 36 months prior to manuscript submission. A.H. Maitland-van der Zee declares unrestricted research grants from Vertex and Boehringer Ingelheim, and is the principal investigator of a Precision Medicine for more Oxygen (P4O2) public private partnership sponsored by Health Holland involving cash or in kind contributions from Boehringer Ingelheim, Breathomix, Fluidda, Ortec Logiqcare, Philips, Quantib-U, Smartfish, SODAQ, Thirona, TopMD and Novartis; consulting fees paid to their institution by AstraZeneca and Boehringer Ingelheim; and payment or honoraria paid to their institution by GlaxoSmithKline, all in the 36 months prior to manuscript submission. They declare that they are an unpaid member of the Data Safety Monitoring Board of the SOS BPD Study; that they are unpaid president of the Federation of Innovative Drug Research in the Netherlands (FIGON); and that they are president of the European Association of Systems Medicine. G. Brusselle declares payment or honoraria from AstraZeneca, Boehringer Ingelheim, Chiesi, GlaxoSmithKline, Novartis, Sanofi and Teva in the 36 months prior to manuscript submission. G.W. Canonica declares no competing interests. A. Agusti declares no competing interests. R. Faner declares research grant payments from GlaxoSmithKline, Menarini, AstraZeneca, ISC-III and the Spanish National Health Service; consulting fees from GlaxoSmithKline; and payments or honoraria from Chiesi, all in the 36 months prior to manuscript submission. C.F. Vogelmeier declares grant payments to their institution from the German Ministry of Education and Science (BMBF), AstraZeneca, Boehringer Ingelheim, GlaxoSmithKline, Grifols and Novartis; consulting fees from AstraZeneca, Boehringer Ingelheim, CSL Behring, Chiesi, GlaxoSmithKline, Menarini, Novartis and Nuvaira; payments or honoraria from Aerogen, AstraZeneca, Boehringer Ingelheim, CSL Behring, Chiesi, GlaxoSmithKline, Menarini and Novartis, all in the 36 months prior to manuscript submission. M. Nawijn declares unrestricted research grant payments to their institution from Chan Zuckerberg Initiative and GlaxoSmithKline in the 36 months prior to manuscript submission; and that they are coordinator of the Human Cell Atlas Lung Biological Network. M. van den Berge declares research grant payments to their institution from GlaxoSmithKline, AstraZeneca, Novartis, Genentech and Roche in the 36 months prior to manuscript submission. F. Rusconi declares no competing interests. C. Pilette declares no competing interests. V. Ramiconi declares unrestricted educational grant payments to her organisation from Novartis, Pfizer, AstraZeneca, Chiesi, GlaxoSmithKline, AbbVie, LeoPharma, Boehringer Ingelheim, Sanofi, Regeneron, OM Pharma, Merck Sharp \& Dohme, Roche and DBV Technologies, in the 36 months prior to manuscript submission. C. Coleman is an employee of the European Lung 
Foundation. R. Chaudhuri declares funding from 3TR European Union Innovative Medicines Initiative 2 to their institution for the present manuscript. They also declare grant funding from AstraZeneca within a Medical Research Council project; lecture fees from GlaxoSmithKline, AstraZeneca, Teva, Chiesi, Sanofi and Novartis; support for attending conferences from Chiesi, Napp, Sanofi, Boehringer Ingelheim, GlaxoSmithKline and AstraZeneca; and participation on advisory boards for GlaxoSmithKline, AstraZeneca, Teva, Chiesi and Novartis, all in the 36 months prior to manuscript submission. K.F. Chung declares grant payments to their institution from the Medical Research Council (precision medicine for severe asthma), the Engineering and Physical Sciences Research Council (air pollution and asthma) and GlaxoSmithKline (eosinophils); consulting fees from the scientific committee of the Clean Breathing Institute (funded by the GlaxoSmithKline consumer division); speaking fees for engagements from Novartis and AstraZeneca; an educational grant paid to their institution for the 11th London International Cough Symposium from AstraZeneca, Merck, Shionogi, Nocion, Bellus and Bayer; participation on a data safety management board for Nocion (antitussive); and participation on advisory boards for Nocion, Shionogi and Merck (antitussives) and GlaxoSmithKline, Roche and Novartis (asthma and COPD), all in the 36 months prior to manuscript submission. J. Wedzicha declares grant payments to their institution from GlaxoSmithkline, AstraZeneca, Novartis, Genentech, Boehringer Ingelheim and Chiesi; paid participation on a Data Safety Monitoring Board for Virtus; and editorship of the American Journal of Respiratory and Critical Care Medicine, for which an honorarium is paid jointly to them and their institution. S. Saglani declares no competing interests. M.P. Van der Schee declares consulting fees from Owlstone Medical in the 36 months prior to manuscript submission; and that they hold stock or stock options in Owlstone Medical and Sanome. L. Heaney declares grant payments to their institution from Novartis UK, GlaxoSmithKline, Amgen, Genentech/Hoffmann la Roche, AstraZeneca, Medimmune, Aerocrine and Vitalograph; payments or honoraria from Novartis, Genentech/Hoffmann la Roche, Sanofi, Evelo Biosciences, GlaxoSmithKline, AstraZeneca, Teva, Theravance and Circassia; support for attending meetings from AstraZeneca, Boehringer Ingelheim, Chiesi, GlaxoSmithKline and Napp; and participation on advisory boards for Novartis, Genentech/Hoffmann la Roche, Sanofi, Evelo Biosciences, GlaxoSmithKline, AstraZeneca, Teva, Theravance and Circassia, all in the 36 months prior to manuscript submission. A. Bourdin declares unrestricted grant payments from AstraZeneca and Boehringer Ingelheim; advisory board fees from AstraZeneca, GlaxoSmithKline, Regeneron, Sanofi, Novartis, Chiesi, Amgen; lecture payments from AstraZeneca, GlaxoSmithKline, Regeneron, Sanofi, Novartis, Chiesi and Amgen; and support for attending meetings from AstraZeneca, GlaxoSmithKline, Regeneron, Sanofi and Novartis, all in the 36 months prior to manuscript submission. G. Roberts declares funding from 3TR European Union Innovative Medicines Initiative 2 to their institution for the present manuscript. R. Djukanovic declares consulting fees from Synairgen plc in the 36 months prior to manuscript submission; that they are chair of the European Respiratory Society Clinical Research Collaboration on severe asthma (SHARP); and that they hold shares in Synairgen plc. P. Kuna declares lecture fees from Adamed, AstraZeneca, Boehringer Ingelheim, Berlin Chimie, Menarini, Chiesi, HAL Allergy, Lekam, Mylan, Novartis, Polpharma and Teva in the 36 months prior to manuscript submission. M. Kupczyk declares funding from 3TR European Union Innovative Medicines Initiative 2 for the present manuscript. They also declare payment or honoraria from AstraZeneca, Chiesi, GlaxoSmithKline, Novartis, Lekam, Alvogen, Emma, Nexter and Berlin Chemie in the 36 months prior to manuscript submission. J. Axmann declares no competing interests. H. Staudinger declares that they are an employee of Sanofi Genzyme; and that they hold stock or stock options in Sanofi and Merck. G.W. Clarke declares that they are an employee of AstraZeneca; and that they hold stock or stock options in AstraZeneca. S.E. Dahlen declares consulting fees from AstraZeneca, Caiman Chemicals, GlaxoSmithKline, Merck, Novartis, Regeneron, Sanofi and Teva, outside the present manuscript. C. Brightling declares support from 3TR European Union Innovative Medicines Initiative 2 for the present manuscript. They also declare grant payments to their institution from GlaxoSmithKline, AstraZeneca, Novartis, Chiesi, Sanofi, Genentech, Merck, Mologic, 4DPharma and Gossamer; consulting fees paid to their institution from GlaxoSmithKline, AstraZeneca, Boehringer Ingelheim, Novartis, Chiesi, Sanofi, Genentech, Merck, Mologic, 4DPharma, Gossamer, TEVA, Regeneron, Roche and CSL Behring, all in the 36 months prior to manuscript submission.

Support statement: This project has received funding from the Innovative Medicines Initiative 2 Joint Undertaking (JU) under grant agreement number 831434 (3TR). The JU receives support from the European Union's Horizon 2020 research and innovation program and EFPIA. The content of this publication reflects only the authors' view and the $\mathrm{JU}$ is not responsible for any use that may be made of the information it contains. Funding information for this article has been deposited with the Crossref Funder Registry.

\section{References}

1 Global Initiative for Asthma. Global Strategy for Asthma Management and Prevention. 2020.

2 GOLD Committee. 2021 Global Strategy for Prevention, Diagnosis and Management of COPD, 2021.

3 Holguin F, Cardet JC, Chung KF, et al. Management of severe asthma: a European Respiratory Society/ American Thoracic Society Guideline. Eur Respir J 2020; 55: 1900588.

$4 \quad$ George L, Brightling CE. Eosinophilic airway inflammation: role in asthma and chronic obstructive pulmonary disease. Ther Adv Chronic Dis 2016; 7: 34-51. 
5 Yousuf A, Brightling CE. Biologic drugs: a new target therapy in COPD? COPD J Chronic Obstr Pulm Dis 2018; 15: 99-107.

6 Agache I, Lau S, Akdis CA, et al. EAACI Guidelines on Allergen Immunotherapy: house dust mite-driven allergic asthma. Allergy 2019; 74: 855-873.

7 Menzies-Gow A, Bafadhel M, Busse WW, et al. An expert consensus framework for asthma remission as a treatment goal. J Allergy Clin Immunol 2020; 145: 757-765.

8 Kavanagh JE, d'Ancona G, Elstad M, et al. Real-world effectiveness and the characteristics of a "super-responder" to mepolizumab in severe eosinophilic asthma. Chest 2020; 158: 491-500.

9 Eger K, Kroes JA, Brinke A, et al. Long-term therapy response to anti-IL-5 biologics in severe asthma-a real-life evaluation. J Allergy Clin Immunol Pract 2020; 9: 1194-1200.

10 Felson DT, Smolen JS, Wells G, et al. American College of Rheumatology/European League Against Rheumatism provisional definition of remission in rheumatoid arthritis for clinical trials. Arthritis Rheum 2011; 63: 573-586.

11 Wailoo A, Hock ES, Stevenson M, et al. The clinical effectiveness and cost-effectiveness of treat-to-target strategies in rheumatoid arthritis: a systematic review and cost-effectiveness analysis. Health Technol Assess 2017; 21: 1-258.

12 Lefaudeux D, De Meulder B, Loza MJ, et al. U-BIOPRED clinical adult asthma clusters linked to a subset of sputum omics. J Allergy Clin Immunol 2017; 139: 1797-1807.

13 Hekking PP, Loza MJ, Pavlidis S, et al. Pathway discovery using transcriptomic profiles in adult-onset severe asthma. J Allergy Clin Immunol 2017; 141: 1280-1290. 